\section{Floor Disinfection in the United States}

\section{To the Editor:}

As you know, the Germans are very great in disinfection. The strong companies and many hygien ists still recommend routine floor disinfection with mostly aldehydes. We, as many others, found no influence of floor disinfection on the incidence of hospital acquired infections even in intensive care units.

National health authorities in Great Britain, Netherlands, Danmark, Sweden and the WHO do not recommend routine disinfection of hospital floors except immediately after spoiling with blood, urine sputum or other infectious secretions. What is the current recommendation in the USA?

DR. FRANZ DASCHNER Freiburg. Germany

Dr. Daschner's letter was referred to Dr. Willson Fahlberg and George F. Mallison for their replies.

In principle, most hospital authorities do not recommend routine disinfection of hospital floors, as there is no direct evidence correlating any particular level of contamination with an increased risk of infection. However, the American Public Health Association has recommended standards for acceptable levels of contamination of hospital floors (Health Lab Sci 7:3 1970). Rodac plate counts of 25 or less colonies is considered good, 26-50 fair and more than 50 poor. The American College of Surgeons in the Manual on Control of Infection in Surgical Patients (edited by W. A. Altmeier et al, JB Lippincott Co, 1976) states that "the bacteriological count on the unused surface of the floor, 12 hours after cleaning and disinfecting, should be less than five organisms per square centimeter." They cite the detergent germicides as being very effective in both cleaning and disinfecting. The Centers for Disease Control Manualon Isolation Techniques for Use in Hospitals (second edition, 1975) discusses floor disinfection as concurrent or terminal in the context of patient rooms or other areas where patients with proven infectious diseases have been isolated. The American Hospital Association Manual on Infection Control in the Hospital (3rd edition) states that, "Environmental sanitation is not believed to have as its primary purpose a direct antibacterial effect by the cleaning agents themselves; its main purpose is to physically remove microorganisms from the various fomites that might transmit them to patients." The 1981 accreditation manual for hospitals (JCAH, Chicago, IIl) requires the development of a hygienic environment for patients and staff and an evaluation of cleaning effectiveness based upon a liaison with the infection control committee and their observations.

All hospitals have a routine of promptly disinfecting any floors that have become grossly contaminated with infectious secretions or excretions. The rooms of patients who have been in isolation for a transmissible infectious disease are both concurrently and terminally cleaned, usually with a detergent disinfectant. Most hospitals routinely clean the floors of all surgical theaters between patients with an appropriate disinfectant detergent regardless of whether the patient had an infectious disease or not. Floors of surgical intensive care and burn units are regularly disinfected. Rooms of patients in reverse (protec(ive) isolation, renal and peritoneal dialysis units and certain other special areas are disinfected daily. In most instances, the disinfecting detergent agent(s) employed are selected specilically for their activity against various microorganisms commonly present in a given area.

WILLSON J. FAHLBERG, PH.D. Department of Microbiology and Immunology Baylor College of Medicine Houston, Texas

There certainly is no epidemiologic justification familiar to me that floor surfaces in patient care facilities must be disinfected in the absence of contamination with potentially infectious material. However, as an aside on a very similar subject, the work by Dr. J. Owen Hendley at the University of Virginia clearly indicates a need for disinfection of hospital surfaces that may be contacted by the hands; showing the possibility that rhinovirus infections may be transmitted by contamination from surfaces, to hands, to nasal or conjunctival mucosa ( $N E n g l$ J Med 288:1361-4, 1973).

Nonetheless, even with the lack of epidemiologic justification for disinfection of hospital floors, we have recommended for some years that disinfectant-detergent products be used routinely on all floors in patient-care areas of hospitals. One of the reasons for this recommendation is that disinfectant-detergent products need not be more expensive than heavy duty, detergent-only products. Further, housekeeping is so labor-intensive in the United States that more than $90 \%$ of total costs are for labor, not for (continued on page 284) 


\section{The Untouchable.}

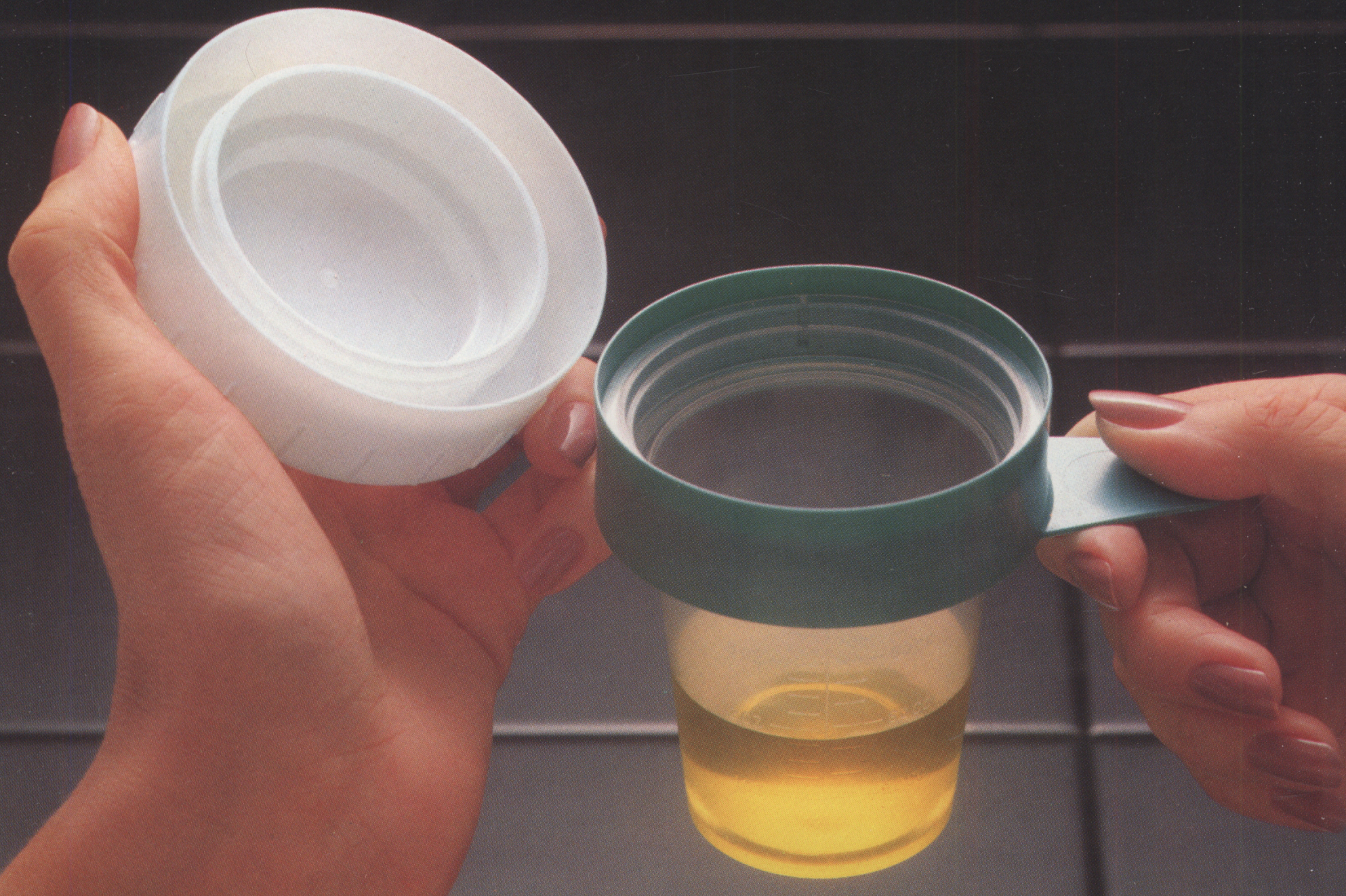




\section{Introducing \\ Asepti-Catch: \\ Designed to be \\ more patient-proof \\ than any other \\ midstream \\ specimen set.}

You know what it's like...

The fumbling, bumbling, balancing act...The grappling with lids and containers that so many patients go through when they try to collect a midstream urine specimen.

And the result - too often, samples contaminated by everything from unsterile surfaces to their own bodies.

Our new Asepti-Catch reduces those problems with an easy-use design so fumble-free, it's practically patient-proof.

Look how easily it works.
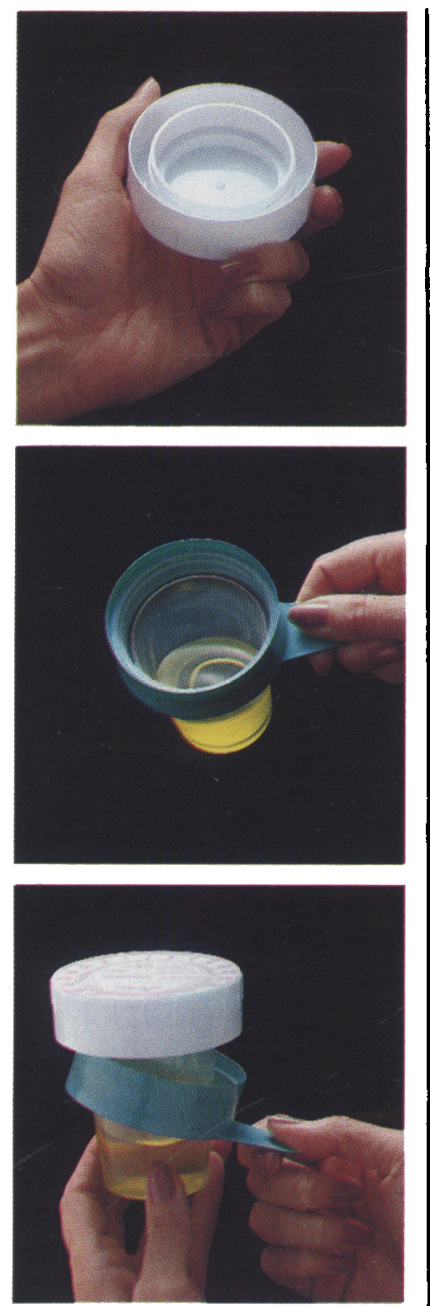

1. This self-contained, recessed cap means you can set the cap down either side up with reduced chance of contamination of sterile surfaces. This unique patented* design also helps to reduce patient touch contamination of the sterile inner surface of the cap. And unlike other midstream sets, there's no need for a protective cover on the cap that requires patient manipulation. 2. An innovative bandle for ease of use is attached to a patented protective collar design that keeps the container rim recessed and clean during the entire collection procedure.

3. The bandle and protective collar can be easily removed by simply pushing downward on the handle after the cap is placed on the container.
Each Asepti-Catch comes with three cleansing towelettes, simple directions and a patient label already affixed to the cap.

Asepti-Catch. The untouchable one. Your patients couldn't handle anything easier.

For a free sample, just contact your American Pharmaseal representative. Or write to American Pharmaseal, Nursing Products Division, P.0. Box 1300, Glendale, CA 91209. 
(continued from page 281)

materials; well under $10 \%$ of total housekeeping costs are for the products used for cleaning and/or disinfecting floors.

If disinfectant-containing products were not used in floor cleaning, it is likely that wet mops would become so contaminated during a work-shift that there might be increased rather than decreased microbial contamination after "cleaning" (and we recommend additionally that the mops be laundered and thoroughly dried daily). Some references cited in our paper "Housekeeping in operating suites," AORN Journal 21:213-220, 1975, indicate that disinfectant-containing products are more effective in reducing microbial contamination than detergents and water alone (T.S. Gable, Hospitals, 40:107-111, February 16, 1966; G.A.J. Ayliffe, B.J. Collins, E.J.L. Lowbury, $\mathrm{Br}$ Med J 2:442-445, 1966; W.D. Foster, Lancet, 1:670-673, 1960; and J.S. Kuipers, J Hygiene, 66:625-631, 1968).

Apparently, use of two cleaning products (one with a disinfecting ingredient and the other with a detergent only) is suggested by Dr. Daschner based on European experience. This policy might increase labor costs of housekeepers and their supervisors because of decisions that would have to be made on how and when a disinfectant-detergent floor cleaning would be justified and dispatched. In my view, such a special cleaning for known presence of potentially infectious material would likely cost more than if a disinfectant-detergent were used for routine cleaning.

In summary, I beleive that it is not a cost disadvantage to use disinfectantdetergent products for all floors in patient-care areas of hospitals.

\section{GEORGE F. MALLSON}

Assistant Director

Bacterial Diseases Division Center for Infectious Diseases Atlanta, Georgia

\section{Detection of Bacteremia: Technical Aspects of the Blood Culture}

\section{To the Editor:}

In the article "Detection of Bacteremia: Technical aspects of the blood culture" (2:399-400, 1981), I would like to respectfully disagree with $\mathrm{Dr}$. Weinstein's statement on subcultures. He comments that subcultures are routine at 7 or 14 days before the blood culture is discarded. While this practice may currently be the case in many hospitals, this practice should now be abandoned. In the past two years, several studies have been done which indicate that the terminal subculture does not detect previously unsuspected bacteremia. Those terminal subcultures that are positive usually appear from patients where other blood cultures were positive earlier. These studies have been documented in standard blood culture media involving over 14,000 blood cultures in a study by Campbell and Washington' and in 2,780 cultures by Gill. $^{2}$ In a study using the BACTEC radiometric blood culture media, Araj et al could not demonstrate any significant value of terminal subcultures in 5,354 blood culture bottles. ${ }^{3}$ This laboratory practice should be abandoned. The aforesaid, however, does not denigrate the value of subcultures within the first 24 hours.

\section{REFERENCES}

1. Campbell J, Washington JA Jr: Evaluation of the necessity for routine terminal subcultures of previously negative blood cul. tures. J Clin Microbiol, 12:576-578, 1980.

2. Gill VJ: Lack of clinical relevance in routine terminal subculturing of blood cultures. J Clin Microbiol, 14:116-118, 1981.

3. Araj GF, Hopfer RL, Wenglar M. et al: Value of terminal subcultures from negative BACTEC blood culture bottles. J Clin Microbiol, 14:589-590, 1981.

PETER A. GROSS, M.D. Director of Internal Medicine Professor of Medicine New Jersey Medical School Hackensack, New Jersey

Dr. Melvin Weinstein responds to Dr. Gross' comments below.

Dr. Gross correctly points out that three recent studies have demonstrated no advantage to routine terminal subculture after seven days incubation. ${ }^{1-3}$ One study reported observations made during a three-week study period' and another examined results during a three-month time span. ${ }^{3}$ These somewhat limited evaluations may not be adequate to support Dr. Gross' conclusion that the practice of performing terminal subcultures be abandoned completely. Indeed, Araj et al ${ }^{3}$ concluded their report by recommending that laboratories review their terminal subculture results before making changes in blood culture policy. In at least one institution where this recommendation was followed, the microbiologist decided to continue performing terminal subcultures. $^{4}$

Campbell and Washington suggest that seven days incubation of blood cultures is probably adequate for general hospitals but that a second week of incubation is indicated in suspected endocarditis, persistent or recurrent infection, and in laboratories which serve referral centers.' Reller also has recommended a two-week incubation of blood cultures in suspected endocarditis. 5 To my knowledge there are no published data on the value of terminal subcultures at the end of a two-week incubation period. During a 21-month period (1975-1977) at the University of Colorado Health Sciences Center (UCHSC), more than 15,000 blood cultures were obtained of which 1069 (7\%) were positive for microorganisms which represented true bacteremia as determined clinically by members of the Infectious Disease Service (Weinstein MP, Reller LB, unpublished data). Fifty-three microorganisms, representing $5 \%$ of all clinically important isolates, were detected only by the terminal subculture at 14 days. Since 1977 the clinical microbiology laboratory at UCHSC has continued to identify $5 \%$ of clinically important isolates, in particular gonococci, cryptococci, and Candida $s p p$., only by the terminal subculture (Reller LB, personal communication). While the yield is limited, the accompanying table shows that the microorganisms isolated represent a broad spectrum of human pathogens.

Is the limited yield worth the extra cost and effort? Laboratory directors hopefully in consultation with interested clinicians, will have to judge the relative value of terminal subcultures in their institutions. At our teaching hospital all blood cultures are incubated for 14 days, and terminal subcultures are and will continue to be performed.

Lastly, Dr. Gross' letter confuses data from two of the studies he quotes. Campbell and Washington ${ }^{1}$ evaluated (continued on page 286) 